\title{
Finite Element Assisted Numerical Comparison of Single and Two Phase Inductively Coupled Power Transfer Systems*
}

\author{
Pratik Raval $^{\#}$, Dariusz Kacprzak, Aiguo Patrick Hu \\ Department of Electrical and Computer Engineering, The University of Auckland, Auckland, New Zealand. \\ Email: "prav010@aucklanduni.ac.nz
}

Received May $13^{\text {th }}, 2013$; revised June $13^{\text {th }}, 2013$; accepted June $21^{\text {st }}, 2013$

Copyright (C) 2013 Pratik Raval et al. This is an open access article distributed under the Creative Commons Attribution License, which permits unrestricted use, distribution, and reproduction in any medium, provided the original work is properly cited.

\begin{abstract}
Inductively coupled power transfer systems (ICPT) are becoming ubiquitous in industry. Many such systems are excited with single or multi-phase input current. This leads to increased complexity in comparing such systems when solely using the magnetic frequency analysis. This paper utilizes modern finite element method analysis software to propose a novel software methodology for the numerical comparison of single and two phase ICPT systems as demonstrated on a three dimensional (3D) battery charging system. The sinusoidal magnetic frequency response of a single phase system is compared to the magnetic transient response of a multi-phase current system by use of a novel software methodology proposed in this paper. This consists of a transient response analysis to determine compute the resulting magnetic response over the duration of an input current period on the two phase system. The resulting non-sinusoidal response is then integrated over a whole period to extract the root-mean-square value for comparison with that of a single phase system across a 3D cubic power zone.
\end{abstract}

Keywords: Finite Element Method; Electromagnetics; Magnetics; Induction; Wireless-Power-Transfer

\section{Introduction}

The technique termed inductively coupled power transfer (ICPT) is a wireless-power-transfer technique that transfers power across an air-gap by means of magnetic induction [1]. This technique removes the inconveniences caused from physical wires by providing power in hard to reach places where conventional direct electrical connections are inconvenient, hazardous, or impossible, lowers maintenance requirements as there is less wear and tear from wet, dirty, moisturized and hazardous environments, and provides enhanced safety as it is free of sparking and can be used in potentially explosive atmospheres and supports freedom of mechanical movement of any load(s) as opposed to a localized load(s). Naturally, this has led to many ICPT applications including monorail systems [2,3], people mover or transportation applications such electric cars, trains and buses [4], biomedical implantation and more recently low power consumer battery charging applications [5]. However, a majority of current such applications largely only support unidirec-

\footnotetext{
*The author declares no conflict of interest.

"Corresponding author.
}

tional and/or bidirectional load movement. That is, there is no support for three-dimensional (3D) load movement along orthogonal axes. This is due to a lack of generation of an omnidirectional primary link that provides a 3D power transfer window. This paper uses the developed 3D cage-like primary magnetic structures that compose a unique 3D ICPT system. Furthermore, depending on the application requirements, the primary AC excitation currents in these systems may be applied as single or multiphase. For analysis, such excitation of primary currents must be numerically computed using advanced modern finite element method (FEM) analysis software as in [6]. The FEM software being used is JMAG designer.

This paper proposes a methodology for the numerical comparison of a single and two phase 3D battery charging ICPT system in terms of the resulting magnetic field.

\section{Proposed ICPT System}

The battery charging system operating at $155 \mathrm{kHz}$ is illustrated in Figure 1. The power converter inverts a DC input to distribute an AC power transfer window through the primary track coils. Typically, the power converter 


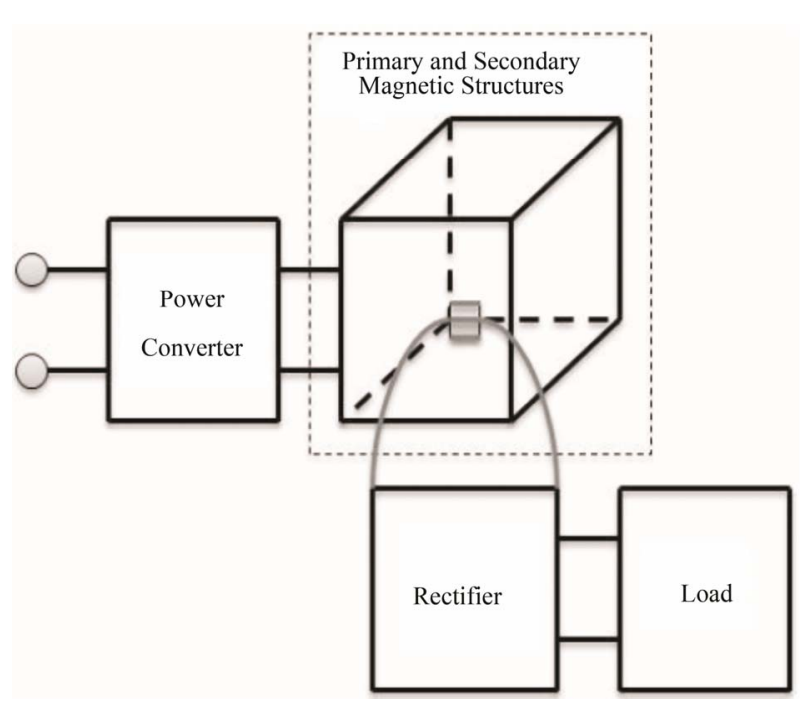

Figure 1. Battery charging ICPT system.

may consist of a primary compensation network with the purpose of providing a resonant AC current. This current is fed into the primary magnetic winding structure. The objective of the primary magnetic structure is to distribute magneto-motive force (MMF) uniformly throughout the entire cubic power transfer volume. The secondary magnetic structure, often termed a pick-up, induces an AC voltage. This voltage is often weak and noisy. So, typically pick-up compensator networks are used to make the induced signal stronger. The result is rectified before operating a load. The presented single and two phase systems are excited with an equivalent ampere-turns ratio [7]. This ensures that an equal MMF is input in ampere current-turns to produce the same magnetic flux density within a pre-defined cubic power transfer volume of $1920 \mathrm{~cm}^{3}$. The only differences between the two systems presented next are the orientation of the primary winding structures and the input phase of the primary excitation currents.

\section{Single Phase System}

The single phase system is shown in Figure 2. This system consists of several over-layed planar rectangular winding structures that are vertically displaced. This generates a predominant normal or vertical component in magnetic flux density. The winding structures are surrounded by a ferromagnetic material casing. This ferrite material acts as a magnetic circuit with three main functions. Firstly, to provide a low magnetic reluctance pathway to the magnetic flux vectors to guide the flux through the ferrite and then disperse the field back into the intended power zone. Secondly, to confine the magnetic flux thereby reducing the flux vectors leaked outwards in an effort to reduce flux leakage so as to reduce
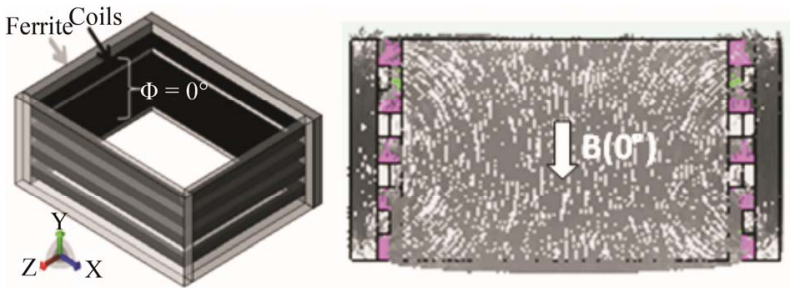

Figure 2. Single phase system (Left) and MFD vectors (Right).

potentially harmful electromagnetic interference. Thirdly, to enhance the field with a high relative permeability of 1200. In this single phase system, the AC sinusoidal excitation primary currents are applied simultaneously and in phase to the primary track coils. This produces a net vertically directed with the simulated magnetic flux density (MFD) distribution vectors also shown in Figure 2.

\section{Two Phase System}

The two phase system is shown in Figure 3. This system consists of two Helmholtz coil pairs along orthogonal axes. The first Helmholtz coil pair is input with an AC sinusoidal primary current that is in phase but of opposite polarity. The second Helmholtz coil pair is similarly input with AC sinusoidal primary current that has a 90 degrees phase difference to the current applied in the first Helmholtz coil pair. That is, the primary currents are applied sequentially and in phase quadrature to parallel but adjacent coil windings so as to produce an elliptical rotating magnetic field resulting from fields $B\left(0^{\circ}\right)$ and $B\left(90^{\circ}\right)$. The resulting winding structures are surrounded by ferromagnetic material casing. In this case, the ferrite acts as an electromagnetic attractor that essentially attracts the flux from unlike magnetic poles. This aids the propagation of the magnetic field from one side of the box to the other that in essence distributes the flux throughout the 3D cubic power transfer volume. The resulting instantaneous MFD distribution vectors are also shown in Figure 3.

\section{System Comparison}

The numerically computed parameter of comparison $B_{R M S}$ is shown below. This is defined as the rootmean-square (RMS) value of the averaged magnitude magnetic flux density $|B(a v g)|$ throughout the cubic intended power zone and $N$ is the number of steps in the transient response. The intended power region to computing the cubic average is illustrated in Figure 4.

$$
B_{R M S}=\sqrt{\frac{1}{N} \sum_{i=1}^{N} \mid\left. B_{i}(\text { avg })\right|^{2}}
$$

For a single phase system, $B_{R M S}$ is readily derived 

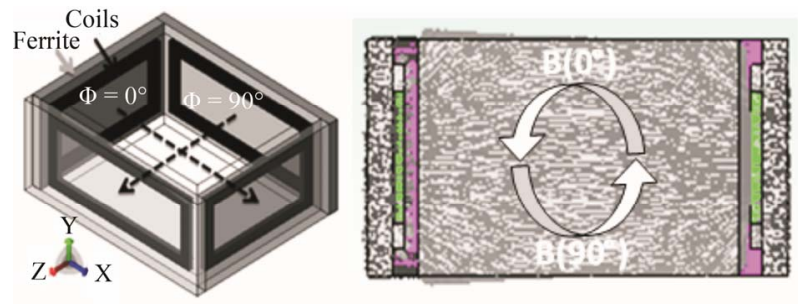

Figure 3. Two phase system (Left) and MFD vectors (Right).

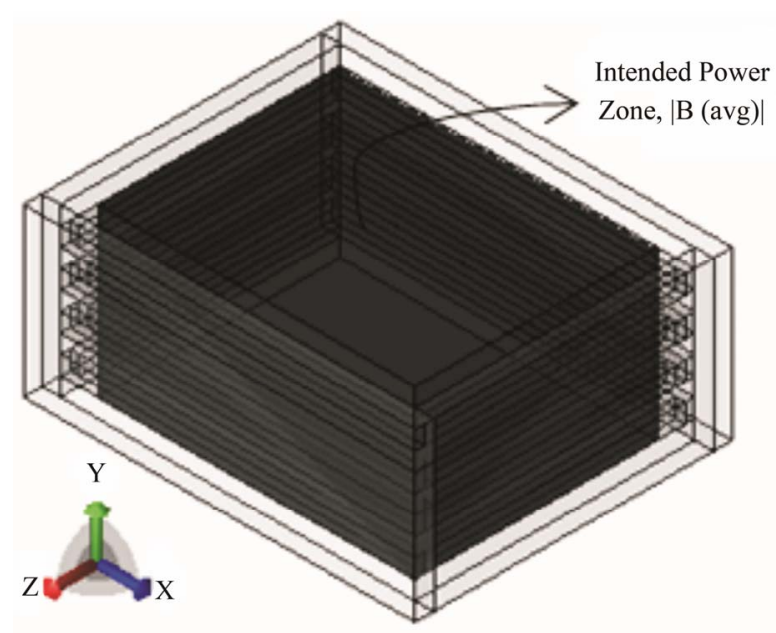

Figure 4. Intended power zone: Cubic volume.

via the simulated magnetic frequency responses instanttaneous peak due to the sinusoidal nature. However, for a two phase system this parameter is no longer sinusoidal so a transient response system model as a simulation methodology is developed as in Figure 5. Firstly, a transient response analysis is performed on the two phase model consisting of 400 steps corresponding to time intervals of 32.25805 ns. Next, the cubic averaged MFD is computed in the cubic volume. Finally, an RMS integrator is performed on the resulting signal, shown in Figure 6, to attain the parameter of comparison $B_{R M S}$.

The resulting comparison of $B_{R M S}$ is shown in Table 1. This result shows that the single phase system is $5.8 \%$ more efficient at distributing the MMF throughout the intended cubic power transfer volume. It is expected that this novel numerical FEM computable comparison technique may be applied to other ICPT systems and multiphase systems. More specifically, this methodology of comparison overcomes a limitation in the FEM software by manual determination of the net peak electromagnetic wave in a multi-phase system that exhibits a non-sinusoidal response over a 3D power transfer volume.

\section{Conclusion}

This paper has proposed a novel FEM software methodology for the numerical comparison of single and two

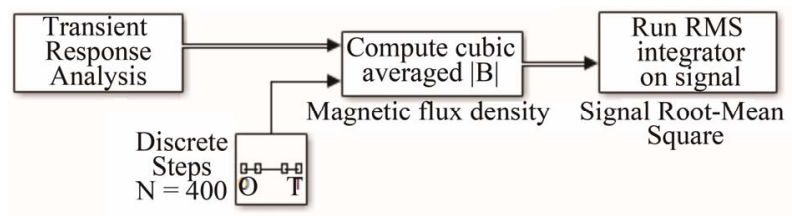

Figure 5. Two phase system software methodology.

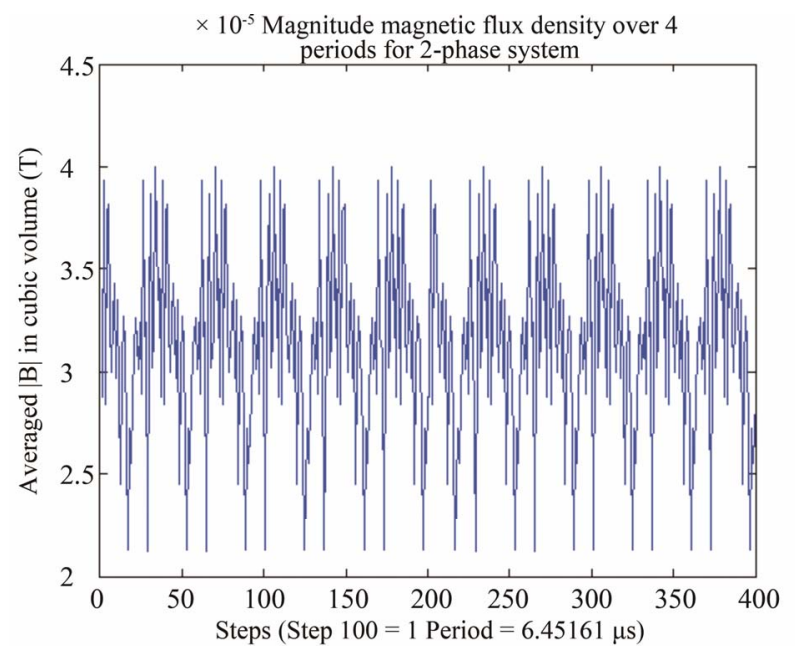

Figure 6. Transient Response of Two Phase System.

Table 1. Comparison of single and two phase ICPT systems.

\begin{tabular}{cc}
\hline ICPT systems input current phase: & $B_{\text {RMS }}(\mu \mathrm{T}):$ \\
\hline Single phase & 33.590 \\
Two phase & 31.745 \\
\hline
\end{tabular}

phase ICPT systems via a transient response analysis where the resulting signal is not of sinusoidal nature over a $3 \mathrm{D}$ power transfer volume in terms of a performance governing metric.

\section{REFERENCES}

[1] E. Abel and S. Third, "Contactless Power Transfer-An Exercise in Topology,” IEEE Transactions on Magnetics, Vol. 20, No. 5, 1984, pp. 1813-1815. doi:10.1109/TMAG.1984.1063160

[2] G. A. J. Elliott, G. A. Covic, D. Kacprzak and J. T. Boys, "A New Concept: Asymmetrical Pick-Ups for Inductively Coupled Power Transfer Monorail Systems,” IEEE Transactions on Magnetics, Vol. 42, No. 10, 2006, pp. 33893391.

[3] D. Kacprzak, "A Novel S-Pickup for High Power Inductive Power Transfer Systems,” IEEE International Magnetics Conference, San Diego, 8-12 May 2006, p. 204.

[4] H. Sakamoto, K. Harada, S. Washimiya, K. Takehara, Y. Matsuo and F. Nakao, "Large Air-Gap Coupler for Inductive Charger for Electric Vehicles,” IEEE Transactions on 
Magnetics, Vol. 35, No. 5, 1999, pp. 3526-3528.

$\underline{\text { doi: } 10.1109 / 20.800578}$

[5] J. M. Barnard, J. A. Ferreira and J. D. van Wyk, "Sliding Transformer for Linear Contactless Power Delivery," IEEE Transactions on Magnetics, Vol. 44, No. 6, 1997, pp. 774-779.

[6] D. Kacprzak, M. J. Gustafsson and M. P. Taylor, “A Finite Element Method Approach to the Design Process of an Aluminum Reduction Cell,” IEEE International Magnetics Conference, IEEE Transactions on Magnetics, San Diego, 8-12 May 2006, p. 668.

[7] O. H. Stielau and G. A. Covic, "Design of Loosely Coupled Inductive Power Transfer Systems," Proceedings of International Conference on Power Systems Technology, Perth, 4-7 December 2000, pp. 85-90. 